\title{
SYMMETRIC CONNECTIVITY WITH MINIMUM POWER CONSUMPTION IN RADIO NETWORKS
}

\author{
G. Călinescu*, I.I. Măndoiu ${ }^{\dagger}$, and A. Zelikovsky ${ }^{\ddagger}$ \\ * Department of Computer Science, Illinois Institute of Technology, Chicago, IL 60616. \\ Research partially done while visiting the Department of Combinatorial Optimization of \\ University of Waterloo, where supported by a NSERC grant. \\ $\dagger$ Department of Computer Science \& Engineering, UC San Diego, La Jolla, CA 92093 \\ $\ddagger$ Department of Computer Science, Georgia State University, Atlanta, GA 30303. Re- \\ search partially supported by NSF Grant CCR-9988331, MRDA and CRDF Award No. \\ MM2-3018 and by the State of Georgia's Yamacraw Initiative. \\ calinesc@cs.iit.edu, mandoiu@cs.ucsd.edu, alexz@cs.gsu.edu
}

\begin{abstract}
We study the problem of assigning transmission ranges to the nodes of a multi-hop packet radio network (also known as static ad hoc wireless network) so as to minimize the total power consumed under the constraint that enough power is provided to the nodes to ensure that the network is connected. Precisely, we require that the bidirectional links established by the transmission range of every node form a connected graph. We call this problem Min-POWER SYMMETRIC CoNNECTIVITY.

Implicit in previous work on transmission range assignment under asymmetric connectivity requirements is the proof that MIN-Power SYMmETRIC CoNNECTIVITY is NP-hard and that a simple algorithm has approximation ratio of 2 . In this paper we establish the similarity between Min-Power SYmmetric ConNECTIVITY and the classic STEINER TREE problem in graphs, give a polynomial-time approximation scheme with performance ratio approaching $1+\ln 2 \approx 1.69$, and present a more practical $15 / 8$ approximation algorithm. We also show that the related MiN-POWER SYMMETRIC UNICAST problem can be solved efficiently by a shortest-path computation in an appropriately constructed graph.
\end{abstract}

\section{Introduction}

Ad hoc wireless networks have received significant attention in recent years due to their potential applications in battlefield, emergency disaster relief, and other application scenarios (see, e.g., $[1,4,5,7,9,11,14,17,16])$. Unlike wired 
networks or cellular networks, no wired backbone infrastructure is installed in ad hoc wireless networks. A communication session is achieved either through single-hop transmission if the recipient is within the transmission range of the source node, or by relaying through intermediate nodes otherwise. We assume that omnidirectional antennas are used by all nodes to transmit and receive signals. Thus, a transmission made by a node can be received by all nodes within its transmission range. This feature is extremely useful for energyefficient multicast and broadcast communications.

For the purpose of energy conservation, each node can (possibly dynamically) adjust its transmitting power, based on the distance to the receiving node and the background noise. In the most common power-attenuation model [12], the signal power falls as $\frac{1}{r^{\kappa}}$ where $r$ is the distance from the transmitter antenna and $\kappa$ is a real constant dependent on the wireless environment, typically between 2 and 4 . Assume that all receivers have the same power threshold for signal detection, which is typically normalized to one. With this assumption, the power required to support a link between two nodes separated by a distance $r$ is $r^{\kappa}$. A crucial issue is how to find a route with minimum total energy consumption for a given communication session. This problem is referred to as Minimum-Energy Routing in [14, 17]. Having every link established in both directions simplifies the one-hop transmission protocols by allowing acknowledgement messages to be sent back for every packet (see, for example [15]). This motivates the study of the Min-Power Symmetric ConNeCTIVITY problem, where a link is established only if both nodes have transmission range at least as big as the distance between them, and the goal is to ensure that the network is connected.

Formally, given a set of points $V$ (representing the nodes in the network) in $E^{2}$ (the two-dimensional Euclidean space) or in $E^{3}$ (the three-dimensional Euclidean space), a transmission range assignment (or range assignment, for short) is a function $r: V \rightarrow R_{+}$. A unidirectional link from node $u$ to node $v$ is established under the range assignment $r$ if $r(u) \geq\|u v\|$, where $\|u v\|$ denotes the Euclidean distance between $u$ and $v$. A bidirectional link $u v$ is established under the range assignment $r$ if $r(u) \geq\|u v\|$ and $r(v) \geq\|u v\|$. Let $B(r)$ denote the set of all bidirectional links established between pairs of nodes in $V$ under the transmission range $r$. In this paper we study the following problem:

Min-Power Symmetric Connectivity: Given a set of nodes $V$ and $\kappa \geq 1$, find a transmission range assignment $r: V \rightarrow R_{+}$minimizing $\sum_{v \in V} r(v)^{\kappa}$ subject to the constraint that the graph $(V, B(r))$ is connected.

Implicit in the work of Clementi, Penna, and Silvestri [5] is a proof that Min-Power Symmetric ConNeCTIVITY in $E^{2}$ is NP-Hard (radio "bridges" in canonical form gadgets, see Definition 3 on page 10 of [5], can be made to be bidirectional links). Therefore, we search for polynomial-time approximation algorithms for this problem. The performance ratio of an approximation algorithm $A$ for a minimization problem is the supremum, over all possible instances $I$, of the ratio between the cost of the output of $A$ when running on $I$ and the cost of an optimal solution for $I$ (the smaller the performance ratio, 
the better). We say that $A$ is an $\alpha$-approximation algorithm if its performance ratio is at most $\alpha$. A fully polynomial $\alpha$-approximation scheme is a family of algorithms $A_{\varepsilon}$ such that, for every $\varepsilon>0, A_{\varepsilon}(1)$ has performance ratio at most $\alpha+\varepsilon$, and (2) runs in time polynomial in the size of the instance and $1 / \varepsilon$.

Kirousis, Kranakis, Krizanc, and Pelc [7] give a minimum spanning tree (MST) based 2-approximation algorithm for MIN-POWER SYMMETRIC CONNECTIVITY (their algorithm is actually designed for a related problem, which we discuss in Section 1.1). We improve the approximation ratio under 2 by exploiting similarities between MIN-PowER SYMMETRIC CoNNECTIVITY and the classic STEINER TREE problem: given an edge-weighted graph $G=(V, E, w)$ and a set $T \subseteq V$ of terminals, find a minimum weight Steiner tree for $T$, i.e., a minimum weight connected subgraph of $G$ which contains $T$. Computing an MST in the complete graph on $T$ with edge-weights equal to the minimum distance in $G$ between corresponding terminals gives a 2-approximation algorithm for Steiner TREe [3, 8]. Zelikovsky [18] gave the first algorithm with approximation ratio less than 2: he used 3-restricted Steiner trees and the concept of gain to obtain an approximation ratio of 11/6. Promel and Steger [10] extend the results of Camerini, Galbiati, and Maffioli [2] and give a polynomial time 5/3-approximation scheme for STEINER TREE, by finding almost optimal 3 -restricted Steiner tree. Zelikovsky [19] gives a polynomial time $(1+\ln 2)$ approximation scheme.

In Section 3 we show that similar concepts can be used for approximating Min-Power Symmetric Connectivity. In particular, we show that the algorithms of [10], [18], and [19] can be modified to give similar approximation ratios for MiN-POWER SYMMETRIC ConNECTIVITY. Our main results are a fully polynomial $1+\ln 2$ approximation scheme based on [19] and a more practical 15/8 approximation algorithm based on [18].

Our algorithms have the same approximation guarantees when network nodes are located in $E^{3}$. In fact, since they work on a graph model of the network, our algorithms can be directly applied to more general problem formulations, e.g., observing given upper-bounds on the transmission range of each node and/or taking into account obstacles that completely block the communication between certain pair of nodes.

In Section 4 we address the related Min-POWER SYMMETric UNICAST problem, which, for given source and destination nodes, $s, t \in V$, asks for a sequence $v_{0}=s, v_{1}, \ldots, v_{k}=t$ of nodes and transmission ranges $r\left(v_{i}\right), i=0, \ldots, k$, under which all bidirectional links $v_{i} v_{i+1}$ are established. We show that MIN-POWER SYMMETRIC UNICAST can be solved efficiently by a shortest-path computation in an appropriately constructed graph.

\subsection{Related Work}

Previous research on symmetric connectivity has addressed only the objective of minimizing the maximum node power $[9,11]$. The objective of minimizing the total power $\sum_{v \in V} r^{\kappa}(v)$ has been addressed under the related 
asymmetric connectivity model, in which unidirectional links give raise to a directed graph on $V$. Four problems have been studied under this model.

First, the ASYMMETRIC UNICAST problem requires establishing a minimum power directed path from a source $s$ to a destination $t$, and is easily solved in polynomial time by shortest-path algorithms.

Second, the ASYMMETRIC BROADCAST problem [14, 17] requires establishing a minimum power arborescence rooted at a given vertex $s$. Clementi et al. [4] prove that ASYMMETRIC BROADCAST is NP-Hard when the nodes are in $E^{2}$. The best known approximation algorithm for ASYMMETRIC BROADCAST [16], based on computing a minimum spanning tree, has performance ratio of at most 12 when the nodes are in $E^{2}$.

Third, in AsYmmetric Multicast, one is given a root $s$ and a set of terminals $T$, and the goal is to establish a minimum-power branching rooted at $s$ which reaches all vertices of $T$. As a generalization of ASYMMETRIC BROADCAST, ASYMMETRIC MULTICAST is also NP-Hard, and based on the work of [16], it is immediate that a minimum Steiner tree would give an approximation ratio of $12 \rho$, where $\rho$ is the approximation for Steiner tree in graphs (the best result known at this moment, given in [13], is $\rho=1+\frac{1}{2} \ln 3+\varepsilon$ ).

Fourth, in the Complete RANGE Assignment problem the objective is establishing a strongly connected subgraph of $V$. Kirousis, Kranakis, Krizanc, and Pelc [7] prove that Complete RANGe Assignment in $E^{3}$ is NP-Hard and, based on the minimum spanning tree, give a 2-approximation algorithm. As opposed to the ASYMMETRIC BROADCAST approximation of [16], the COMPLETE RANGE AssignMENT approximation of [7] is valid in arbitrary graphs (that is, the distance between two points could be arbitrary, not necessarily Euclidean). Clementi, Penna, and Silvestri [5] give an elaborate reduction proving that Complete Range Assignment in $E^{2}$ is also NP-Hard.

The power for the asymmetric COMPLETE RANGE AsSignMENT can be twice less than the power for MIN-POWER SYMMETRIC CONNECTIVITY as illustrated by the following example in which $\kappa=2$. The terminal set (see Figure 1) consists of $n$ groups of $n+1$ points each, located on the sides of a regular $2 n$-gon. Each group has 2 terminals in distance 1 of each other (represented as thick circles in Figure 1) and $n-1$ equally spaced points (dashes in Figure 1) on the line segment between them. It is easy to see that the minimum range assignment ensuring asymmetric connectivity assigns power of 1 to the one thick terminal in each group and power of $\varepsilon^{2}=(1 / n)^{2}$ to all other points in the group. The total power then equals $n+1$. For symmetric connectivity it is necessary to assign power of 1 to all but two thick points, and of $\varepsilon^{2}$ to the remaining points, which results in total power of $2 n-1-1 / n+2 / n^{2}$.

\section{Preliminaries}

We begin by formulating the graph-weighted extension of MIN-POWER SYMMETRIC CoNNECTIVITY. For completeness, we then show that computing an MST gives a 2-approximation for this extension; this result is implicit in the work of Kirousis, Kranakis, Krizanc, and Pelc [7]. 


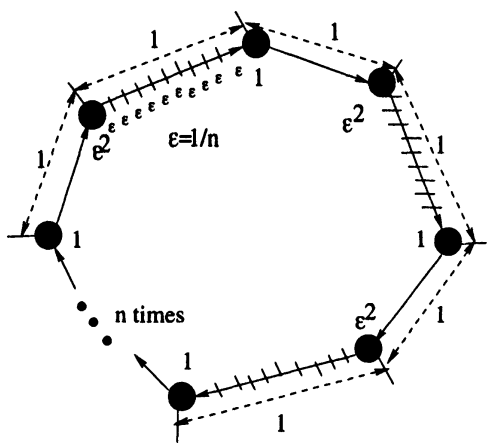

(a)

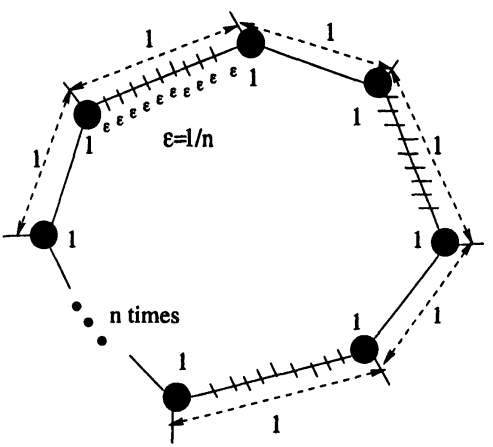

(b)

Figure 1. Total power for the asymmetric Complete RANGe ASSignMent can be twice less than the total power for Min-Power Symmetric Connectivity $(\kappa=2)$. (a) Minimum range assignment ensuring asymmetric connectivity has total power $n+n^{2} \varepsilon^{2}=n+n^{2} \frac{1}{n^{2}}=n+1$. (b) Minimum range assignment ensuring symmetric connectivity has the total power $(2 n-2)+\left(n^{2}-n+2\right) \varepsilon^{2}=2 n-1-\frac{1}{n}+\frac{2}{n^{2}}$.

Let $G=(V, E, c)$ be an edge-weighted graph. For a spanning tree $T=(V, F)$ of $G$, let $\overline{r_{T}}(v)=\max _{u \mid u v \in F} c(u v)$. Define the power-cost of $T$ by

$$
p(T)=\sum_{v \in V} \overline{r_{T}}(v)
$$

Since any connected graph contains a tree, an equivalent formulation of MiNPOWER SYMMETRIC CONNECTIVITY is to ask for a spanning tree with minimum power-cost in the complete graph on $V$ with edge costs given by $c(u v)=$ $\|u v\|^{\kappa}$. Thus, Min-Power SYMmetric ConNeCTIVITY is a special case of the following problem:

Minimum Power-Cost Spanning Tree: Given a connected edge-weighted graph $G=(V, E, c)$, find a spanning tree $T$ of $G$ with minimum power-cost.

All our algorithms work for the this graph-weighted extension of MIN-POWER Symmetric ConNECTIVITY. From now on, we only use this formulation.

Theorem 1 Computing an MST with respect to c gives a 2-approximation for Min-Power Symmetric ConneCTIVITY.

Proof: Let $c(T)=\sum_{u v \in F} c(u v)$. Claim 2 of Theorem 3.2 of [7] is equivalent to

$$
p(T)=\sum_{v \in V} \max _{u \mid u v \in F} c(u v) \leq \sum_{v \in V} \sum_{u \mid u v \in F} c(u v)=2 c(T)
$$

Let $u$ be a vertex incident to an edge of maximum cost. If we root the tree $T$ at $u$, and use $v^{\prime}$ to denote the parent of $v$ in $T$, since $\overline{r_{T}}(v) \geq c\left(v v^{\prime}\right)$ we 


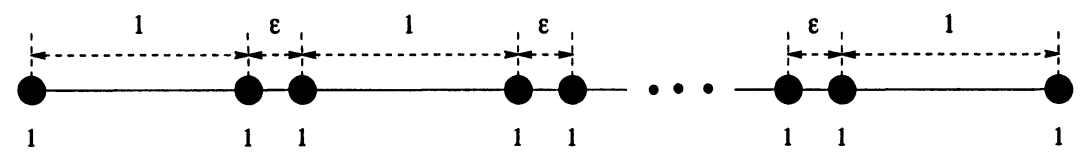

(a)

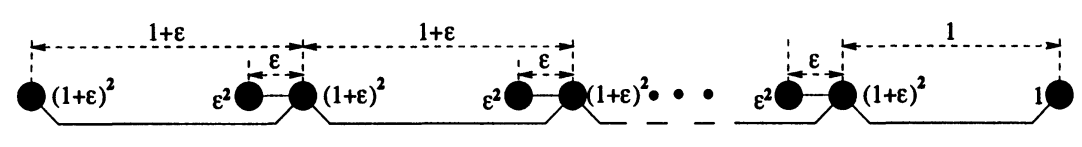

(b)

Figure 2. Tight example for the performance ratio of the MST algorithm $(\kappa=2)$. (a) The MST-based range assignment needs total power $2 n$. (b) Optimum range assignment has total power $n(1+\varepsilon)^{2}+(n-1) \varepsilon^{2}+1 \rightarrow n+1$.

conclude that $p(T) \geq c(T)$. Therefore, if $M S T$ is the minimum spanning tree with respect to $c$ and $O P T$ is the tree with minimum power-cost, we have

$$
p(M S T) \leq 2 c(M S T) \leq 2 c(O P T) \leq 2 p(O P T)
$$

The following example shows that the ratio of 2 given in Theorem 1 is tight. Consider $2 n$ points located on a single line such that the distance between consecutive points alternates between 1 and $\varepsilon<1$ (see Figure 2) and let $\kappa=2$. Then the minimum spanning tree MST connects consecutive neighbors and has power-cost $p(M S T)=2 n$. On the other hand, the tree $T$ with edges connecting each other node (see Figure 2(b)) has power-cost equal $p(T)=n(1+\varepsilon)^{2}+(n-$ 1) $\varepsilon^{2}+1$. When $n \rightarrow \infty$ and $\varepsilon \rightarrow 0$, we obtain that $p(M S T) / p(T) \rightarrow 2$.

\section{3. $\quad k$-Restricted Approach to Symmetric Min-Power Connectivity}

We first give definitions of $k$-restricted decompositions and prove an upper bound on the power-cost of such decompositions. Then we will describe approximation algorithms whose approximation ratios follow from the approximation ratios of Steiner tree algorithms in graphs.

\section{1. $\quad k$-Restricted Decompositions}

A $k$-restricted decomposition $Q$ of the tree $T$ is a partition of $T$ into subtrees $T_{1}, T_{2}, \ldots, T_{p}$ each containing at most $k$ vertices such that each edge of $T$ belongs to exactly one subtree $T_{i}$. The power-cost $p(Q)$ of $Q$ defined to be the 
sum of power-costs of all its elements:

$$
p(Q)=\sum_{T_{i} \in Q} p\left(T_{i}\right)
$$

Theorem 2 For any weighted tree $T$ and any $k \geq 1$, there is a $2^{k}$-restricted decomposition $Q$ of $T$ such that $p(Q) \leq(1+1 / k) p(T)$.

Proof: Without loss of generality we can assume that all edge costs are different. Let the endpoints $r$ and $s$ of the heaviest edge $h$ of $T$ be the roots of $T$, which means that two subtrees of $T-\{h\}$ are rooted at $r$ and $s$, respectively. Then each vertex $v$ of $T$, except $r$ and $s$, has a unique parent. We call the vertices adjacent to $v$, other than the parent of $v$ (if defined), the children of $v$. For each vertex $v$ of $T$, we sort the edges connecting $v$ to its children in increasing order of their cost. For the most costly such edge $e$, we define next $(e)=f$, where $f$ is the edge connecting $v$ to its parent (if $v$ has a parent), or $f=h$ if $v$ does not have a parent. For some other edge $e$ from $v$ to one of its children, we define $\operatorname{next}(e)=e^{\prime}$, where $e^{\prime}$ is the next edge (in the sorted order above) from $v$ to one of its children.

We now construct a rooted directed binary (with arcs going toward the root) tree $B$ as follows. The vertices of $B$ are the edges of $T$ and the root of $B$ is $h$, the heaviest edge of $T$. The $\operatorname{arcs}$ of $B$ consists of $\operatorname{arcs}(e, n e x t(e))$ for each edge $e$ of $T$. It is immediate that every vertex $e=u v$ of $B$ has at most two incoming arcs. Indeed, if $e=r s$, then only the most costly edge of $T \backslash\{e\}$ incident to $r$ and the most costly edge of $T \backslash\{e\}$ incident to $s$, have $e$ as a parent. The other edges of $T e=u v$ have $v$ as the parent of $u$ (the other case being symmetric), and the arcs coming into $e$ are only the most costly edge of $T \backslash\{e\}$ incident to $u$ and the edge in between $v$ and another child of $v$ which precedes $e$ in the sorted order above. Note that each vertex of $B$ has cost since it is an edge of $T$.

Let $B_{i}$ be the set of vertices of $B$ in distance $i$ from the root $h$. There is an integer $0 \leq l<k$ such that $\sum_{j \mid j \equiv l(\bmod k)} c\left(B_{j}\right) \leq \frac{1}{k} c(B)=\frac{1}{k} c(T)$, and let $\bar{B}=\cup_{j \mid j \equiv l \quad(\bmod k)} B_{j}$. Removal of every edge outgoing from $\bar{B}$ decomposes $B$ into subtrees $Q_{i}$ corresponding to subtrees $T_{i}$ of $T$. The number of vertices in $Q_{i}$ is at most $2^{k}-1$ since $Q_{i}$ is a binary tree of height at most $k-1$. Therefore, each $T_{i}$ has at most $2^{k}$ vertices. We denote by $Q$ the $2^{k}$-restricted decomposition of $T$ into $T_{i}$ 's.

Let $e_{i}=\left(v_{i}, u_{i}\right)$ be the root of $Q_{i}$ (note that $e_{i} \in \bar{B}$ ) and, if $e_{i} \neq(r, s)$, rename $v_{i}$ and $u_{i}$ such that $u_{i}$ is the parent of $v_{i}$ in $T$. By the construction of $B$, we have that $\max _{u \mid u u_{i} \in E\left(T_{i}\right)} c\left(u u_{i}\right)=c\left(e_{i}\right)$. Then we have:

$$
p\left(T_{i}\right) \leq c\left(e_{i}\right)+\sum_{v \in V\left(T_{i}\right) \backslash\left\{u_{i}\right\}} \max _{(v, u) \in E(T)} c(v, u) .
$$

For $i \neq j$, the sets $V\left(T_{i}\right) \backslash\left\{u_{i}\right\}$ and $V\left(T_{j}\right) \backslash\left\{u_{j}\right\}$ are disjoint. We conclude that 


$$
\begin{aligned}
p(Q) & =\sum_{i} p\left(T_{i}\right) \\
& \leq \sum_{v \in V(T)} \max _{(v, u) \in E(T)} c(v, u)+\sum_{i} c\left(e_{i}\right) \\
& \leq p(T)+c(\bar{B}) \\
& \leq p(T)+\frac{1}{k} c(T) \\
& \leq\left(1+\frac{1}{k}\right) p(T) .
\end{aligned}
$$

A subtree of $T$ with exactly three edges and two edges is called a fork. So a 3-restricted decomposition $Q$ of $T$ consists of forks and individual edges. We have:

Theorem 3 For any weighted tree $T$, there is a 3-restricted decomposition $Q$ of $T$ such that $p(Q) \leq \frac{7}{4} p(T)$.

Proof: By induction on $n$, the number of vertices of the tree, with the base case obvious. Assume $u v$ is the minimum cost edge in the tree (with $c(u v)=\varepsilon$ ), and let $T_{1}$ and $T_{2}$ be the two trees obtained from $T$ after removing the edge $u v$. Let $c^{\prime}(x y)=c(x y)-c(u v)$, for any edge $x y$ in $T_{1}$ or in $T_{2}$.

By induction, there are 3-restricted decompositions $Q_{1}$ of $T_{1}$ and $Q_{2}$ of $T_{2}$ such that $p^{\prime}\left(Q_{1}\right) \leq \frac{7}{4} p^{\prime}\left(T_{1}\right)$ and $p^{\prime}\left(Q_{2}\right) \leq \frac{7}{4} p^{\prime}\left(T_{2}\right)$. We also assume that $Q_{1}$ and $Q_{2}$ are "fork-maximal", in the sense that no two edges can be merged in fork (such a merging decreases the power-cost). We count the number of forks in $Q_{1}$ and in $Q_{2}$. Assume $T_{i}$ has $n_{i}$ vertices, and $Q_{i}$ has $k_{i}$ forks and $s_{i}$ single edges. Then $n_{i}=2 k_{i}+s_{i}+1$. As $Q_{i}$ is fork-maximal, we also have that $s_{i} \leq 2 k_{i}+1$. Also, $n=n_{1}+n_{2}$. Let $Q$ be the partition of $T$ obtained from $Q_{1}, Q_{2}$, and $u v$. Then we have:

$$
p(Q)=p^{\prime}\left(Q_{1}\right)+p^{\prime}\left(Q_{2}\right)+\varepsilon\left(3 k_{1}+2 s_{1}+3 k_{2}+2 s_{2}+2\right),
$$

while

$$
p(T)=p^{\prime}\left(T_{1}\right)+p^{\prime}\left(T_{2}\right)+\varepsilon n .
$$

So it suffices to show that $3 k_{1}+2 s_{1}+3 k_{2}+2 s_{2}+2 \leq \frac{7}{4} n$, which follows immediately from the the equations on $n, n_{i}$, and $s_{i}$ above.

\subsection{Approximation Algorithms}

Based on the results of [2] or [10] and Theorem 3, it is immediate there exists an algorithm with the approximation ratio $\rho_{3}<\frac{7}{4}+\epsilon$.

Let $\rho_{k}$ be the supremum, over all trees $T$, of the ratio of the power-cost of the minimum power-cost $k$-restricted decompositions to the power-cost of $T$. 


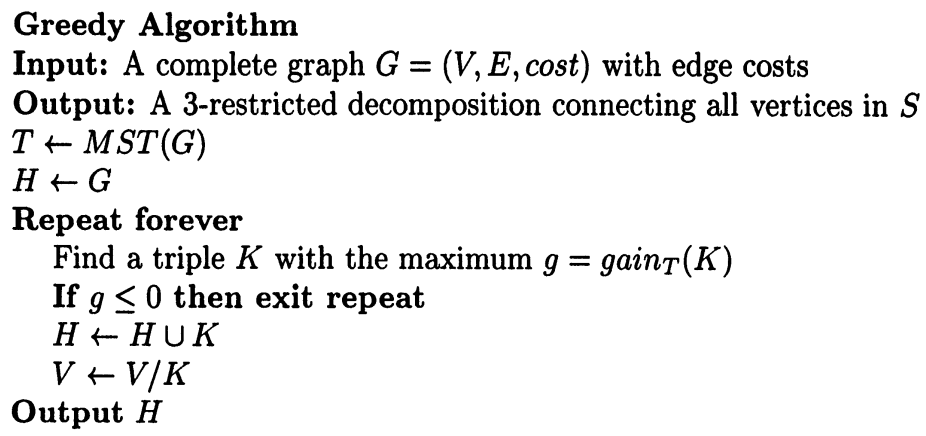

Figure 3. The Greedy Algorithm

Theorem 2 implies that $\rho_{k} \leq 1+\frac{1}{[\lg k]}$, Theorem 3 implies that $\rho_{3} \leq 7 / 4$, and (1) together with the example in Figure 2 imply that $\rho_{2}=2$. In order to obtain better approximation ratios we can approximate the minimum power-cost $k$ restricted decompositions.

In Steiner trees, $k$-restricted decompositions correspond to $k$-restricted Steiner trees. Below we translate the Steiner tree terms into the language of decompositions.

For a set of vertices $V$, denote by $m s t(V)$ the minimum cost of a spanning tree. For a tree $H$ connecting some vertices from $V$, we denote $V / H$ the set of vertices $\mathrm{V}$ after contracting of $H$, i.e., collapsing all vertices of $H$ into a single vertex. Let gain of a subtree $H, \operatorname{gain}(H)$, be

$$
\operatorname{gain}(H)=2 m s t(V)-2 m s t(V / H)-p(H)
$$

It has been proved in [18] that the Greedy Algorithm (see Figure 3) has approximation ratio at most arithmetic mean of $\rho_{2}$ and $\rho_{3}$. Thus we have:

Theorem 4 The Greedy Algorithm for Min-Power Symmetric ConneCTIVITY (see Fig. 3) has approximation ratio of $15 / 8$.

It has been shown in [19] that the $k$-restricted Relative Greedy Algorithm (see Figure 4) has approximation ratio at most $1+\ln 2+\varepsilon$ if $\lim _{k \rightarrow \infty} \rho_{k}=1$.

Theorem 5 The $k$-restricted Relative Greedy Algorithm for MIN-POWER SYMMETRIC CONNECTIVITY (see Fig. 4) has approximation ratio of $1+\ln 2+\varepsilon$.

\section{Minimum Power Symmetric Unicast}

In this section we reformulate MIN-POWER SYMMETRIC UNICAST as a graph problem, and then reduce the latter problem to a single-source single-sink shortest-path computation in an appropriately constructed graph. 
$k$-restricted Relative Greedy Algorithm ( $k$-RGA)

Input: A complete graph $G=(V, E, c o s t)$ with edge costs and an integer $k \leq|V|$

Output: A $k$-restricted decomposition connecting all vertices in $S$

$T \leftarrow M S T(G)$

$H \leftarrow G$

Repeat forever

Find a $k$-restricted tree $K$ with the minimum $r=\frac{p(H)}{2 m s t(V)-2 m s t(V / H)}$

If $r \geq 1$ then exit repeat

$H \leftarrow H \cup K$

$V \leftarrow V / K$

Output $H$

Figure 4. The $k$-restricted Relative Greedy Algorithm ( $k$-RGA).

Min-Power Symmetric Path in Graphs: Given a graph $G=(V, E, c)$ with costs on edges a source $s \in V$ and a destination $t \in V$, find an $s-t$ path in $G$ of the minimum power-cost.

The following example in the Euclidean plane shows that a straightforward application of Dijkstra's algorithm does not work, i.e., a minimum cost $s-t$ path does not always have minimum power-cost. Consider a network consisting of three nodes, $s=(0,3), t=(4,0)$, and $x=(0,0)$, and assume $\kappa=2$. Then the two $s-t$ paths, namely, $(s, t)$ and $(s, v, t)$, have the same cost of 25 but different power-costs: the power-cost of $(s, t)$ is $25+25=50$ while the power-cost of $(s, v, t)$ is $9+16+16=41$.

Our solution of MIN-POWER SYMMETRIC UNICAST first modifies the given graph $G=(V, E, c)$ and then applies Dijkstra's algorithm to the resulted directed graph $G^{\prime}$. We now describe the construction of the directed graph $G^{\prime}=\left(V^{\prime}, E^{\prime}, c^{\prime}\right)$.

For any $u \in V$, we sort all adjacent vertices $\left\{v_{1}, \ldots, v_{k}\right\}$ in ascending order of costs of edges connecting them to $u$, i.e., $c\left(u, v_{i}\right) \leq c\left(u, v_{i+1}\right)$. The vertex $v$ is replaced with a gadget (see Figure 5(a)) as follows:

(i) each edge $\left(u, v_{i}\right)$ is subdivided by a vertex $\left[u, v_{i}\right]$

(ii) for each $u$, we connect all vertices $\left[u, v_{i}\right]$ 's by two directed paths:

$P_{1}=\left(u,\left[u, v_{1}\right], \ldots,\left[u, v_{k-1}\right],\left[u, v_{k-1}\right]\right)$ and

$P_{2}=\left(\left[u, v_{k-1}\right],\left[u, v_{k-1}\right], \ldots,\left[u, v_{1}\right], u\right)$.

(iii) the costs of the $\operatorname{arcs}$ on path $P_{1}$ are $c\left(u, v_{1}\right), c\left(u, v_{2}\right)-c\left(u, v_{1}\right), \ldots$, $c\left(u, v_{k}\right)-c\left(u, v_{k-1}\right)$, respectively, and the cost of all arcs on the path $P_{2}$ is zero.

Finally, each edge $(u, v)$ of $G$ is replaced in $G^{\prime}$ with the two $\operatorname{arcs}([u, v],[v, u])$ and $([v, u],[v, u])$, both of cost $c(u, v)$. Figure $5(\mathrm{~b})$ shows the graph $G^{\prime}$ for the 


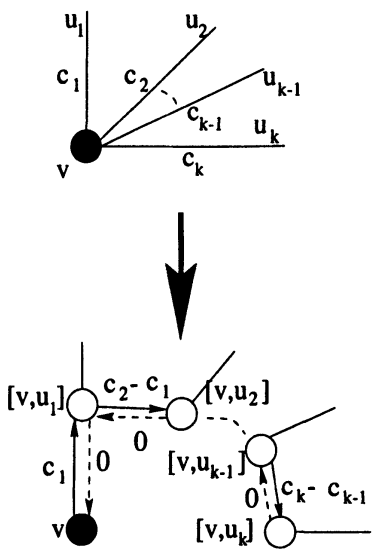

(a)

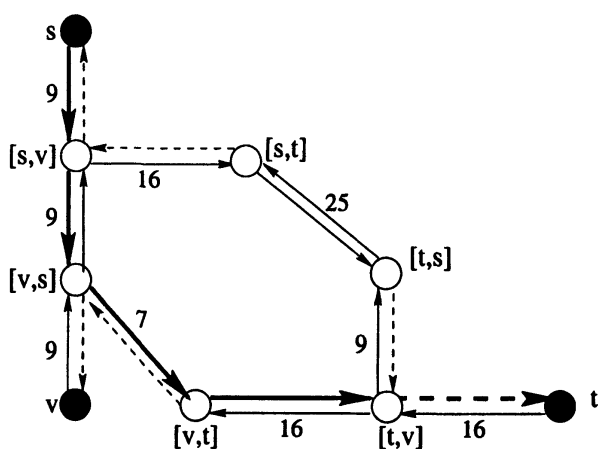

(b)

Figure 5. (a) A vertex $v$ adjacent to $k$ vertices $u_{1}, \ldots, u_{k}$ via edges of cost $c_{1}, c_{2}, \ldots, c_{k}$ and a gadget replacing $v$ with a bidirectional path. The solid edges of the path $\left(v,\left[v, u_{2}\right]\right),\left(\left[v, u_{2}\right]\right),\left[v, u_{3}\right], \ldots,\left(\left[v, u_{k-1}\right],\left[v, u_{k}\right]\right.$ have cost $c_{1}, c_{2}-c_{1}, \ldots$, $c_{k}-c_{k-1}$, respectively. The dashed edges have zero cost. (b) The graph $G^{\prime}$ for the example below. Thick edges belong to the shortest path corresponding to the path $(s, v, t)$ in $G$.

three-node example $s=(0,3), t=(4,0)$, and $x=(0,0)$, with $\kappa=2$. It is easy to see that a shortest $s-t$ path in $G^{\prime}$ corresponds to a minimum power-cost $s-t$ path.

Using the Fibonnaci heaps implementation of Dijkstra's algorithm [6] to compute the shortest $s-t$ path in $G^{\prime}$, and observing that $\left|V^{\prime}\right|=O(|E|)$ and $\left|E^{\prime}\right|=O(|E|)$, we obtain the following

Theorem 6 Symmetric UNICAST is solvable in time $O(|E| \log |V|)$.

\section{Acknowledgements}

The first author thanks Joseph Cheryan, Francisco Zaragoza, and Bhaskar DasGupta for useful discussions in the early stage of this project.

\section{References}

[1] D.M. Blough, M. Leoncini, G. Resta, and P. Santi. On the symmetric range assignment problem in wireless ad hoc networks. In Proc. 2nd IFIP International Conference on Theoretical Computer Science, page (to appear), 2002.

[2] P.M. Camerini, G. Galbiati, and F. Maffioli. Random pseudo-polynomial algorithms for exact matroid problems. Journal of Algorithms, 13:258-273, 1992.

[3] E.-A. Choukhmane. Une heuristique pour le probleme de l'arbre de Steiner. RAIRO Rech. Oper., 12:207-212, 1978. 
[4] A.E.F. Clementi, P. Crescenzi, P. Penna, G. Rossi, and P. Vocca. On the complexity of computing minimum energy consumption broadcast subgraphs. In Symposium on Theoretical Aspects of Computer Science, pages 121-131, 2001, extended version available at http://www.mat.uniroma2.it/ penna/papers/stacs01-TR.ps.gz.

[5] A.E.F. Clementi, P. Penna, and R. Silvestri. On the power assignment problem in radio networks. Electronic Colloquium on Computational Complexity (ECCC), (054), 2000.

[6] T.H. Cormen, C.E. Leiserson, and R.L. Rivest. Introduction to algorithms (2nd ed.). MIT Press, Cambridge, Massachusetts, 2001.

[7] L.M. Kirousis, E. Kranakis, D. Krizanc, and A. Pelc. Power consumption in packet radio networks. Theoretical Computer Science, 243:289-305, 2000.

[8] L. Kou, G. Markowsky, and L. Berman. A fast algorithm for Steiner trees. Acta Informatica, 15:141-145, 1981.

[9] E. Lloyd, R. Liu, M. Marathe, R. Ramanathan, and S.S. Ravi. Algorithmic aspects of topology control problems for ad hoc networks. In Proc. ACM MobiHoc, page (to appear), 2002.

[10] H.J. Promel and A. Steger. A new approximation algorithm for the Steiner tree problem with performance ratio 5/3. Journal of Algorithms, 36:89-101, 2000.

[11] Ram Ramanathan and Regina Hain. Topology control of multihop wireless networks using transmit power adjustment. In INFOCOM (2), pages 404-413, 2000.

[12] T.S. Rappaport. Wireless Communications: Principles and Practices. Prentice Hall, 1996.

[13] G. Robins and A. Zelikovsky. Improved Steiner tree approximation in graphs. In Proceedings of the 11th ACM-SIAM Annual Symposium on Discrete Algorithms, pages 770-779, 2000.

[14] S. Singh, C.S. Raghavendra, and J. Stepanek. Power-aware broadcasting in mobile ad hoc networks. In Proceedings of IEEE PIMRC, 1999.

[15] A.S. Tanembaum. Computer Networks (3rd edition). Prentice Hall, 1996.

[16] P.-J. Wan, G. Calinescu, X.-Y. Li, and O. Frieder. Minimum energy broadcast routing in static ad hoc wireless networks. In Proc. IEEE INFOCOM, vol. 2, pages 1162-1171, 2001.

[17] J.E. Wieselthier, G.D. Nguyen, and A. Ephremides. On the construction of energy-efficient broadcast and multicast trees in wireless networks. In Proc. IEEE INFOCOM, pages 585-594, 2000.

[18] A. Zelikovsky. An 11/6-approximation algorithm for the network Steiner problem. Algorithmica, 9:463-470, 1993.

[19] A. Zelikovsky. Better approximation bounds for the network and Euclidean Steiner tree problems. Technical Report CS-96-06, Department of Computer Science, University of Virginia, 1996. 